\title{
The validity of rasterstereography as a technological tool for the objectification of postural assessment in the clinical and education- al fields: pilot study
}

\author{
Berardi Anna $^{[1]}$, Galeoto Giovanni ${ }^{[2]}$, Tofani Marco ${ }^{[3]}$, Mangone Massimiliano ${ }^{[4]}$, \\ Ratti Serena $^{[1]}$, Danti Arianna ${ }^{[1]}$, Sansoni Julita ${ }^{[2]}$, and Marquez Maria Auxiliadora ${ }^{[5]}$ \\ ${ }^{1}$ Sapienza Università di Roma, Rome, Italy \\ ${ }^{2}$ Department of Public Health and Infectious Diseases, Sapienza University of Rome, Rome, \\ Italy \\ ${ }^{3}$ Neurorehabilitation Unit, Bambino Gesù Children's Hospital, Rome, Italy \\ ${ }^{4}$ Department of Anatomy, Histology, Forensic Medicine and Orthopedics, Sapienza University \\ of Rome, Rome, Italy \\ ${ }^{5} \mathrm{CPO}$ - Paraplegic Center of Ostia Rome, Italy \\ giovanni.galeoto@uniromal.it
}

\begin{abstract}
The present study aimed to validate Formetric 4D with wheelchair users in a sitting position, comparing the results with the data obtained from a postural assessment. Nine individuals with spinal cord injury were evaluated through postural assessment. Each individual was subjected to rasterstereography in a sitting position using the Formetric 4D. Test-retest reliability was evaluated, after four hours: at first, the detection was performed on the same participant three times by three different rater. After four hours, the detection was repeated by the first operator. This study highlighted the ability of Formetric 4D to provide consistent results at different times and with different evaluators, reporting a Cronbach's alpha of 0.74 and excellent intra- and inter-operator stability with an ICC of 0.91 to 0.96 . Compared to the postural evaluation, the degree of accuracy of the measurements acquired with Formetric $4 \mathrm{D}$, analyzed through Spearman's rho, showed statistically significant positive correlations with anthropometric measurements. The present study provides information enabling the use of the Formetric 4D tool in clinical, research, and educational settings this will be a very useful tool that allows students to have a three-dimensional representation of the anatomical components involved in the sitting position, helping them to learn and gain an in-depth understanding of how to perform an objective postural assessment examination.
\end{abstract}

Keywords: Formetric 4D, Posture, Assessment, Spinal Cord Injury.

\section{Introduction}

Seating and postural management describes the way in which our bodies are positioned and managed. People with spinal cord injury, for whom a wheelchair is the means to move and perform their daily activities, often have postural changes due to the absence of mobility and muscle recruitment [1]. An obligatory static posture requires periodic checks to assess and monitor the patient's condition over time to prevent pressure injuries and the aggravation of deformities and avoid tendon and joint retractions [2]. Manual postural assessment is the method currently used by occupational therapists to evaluate these conditions, following the International Organization for Standardization's (ISO) standard number 16840:2013-2018 [3-4] Postural assess- 
ment, however, is a subjective instrument and difficult to understand for students of occupational therapy, because it requires the therapist to record movements or joint deformity through palpation and mobilization. As a result of inadequate professional training, there is a scarcity of rehabilitation professionals experienced in or specially trained to provide seating and mobility recommendations [5]. Although the need to train additional skilled practitioners is clear, the most effective means of training and the tools to evaluate the effectiveness of training programs are not yet clear. Therefore, it is necessary to identify an instrument that provides scientifically valid data for an overall assessment of the patient's posture that can be repeated over time without causing harmful effects to the subject, giving students an instrument that enables them to see in a direct and three-dimensional way the components of the sitting position so that they can compare those components with the results of the postural evaluation. In the literature, several studies have demonstrated the reliability and validity of the rasterstereography performed by the Formetric 4D tool with the patient in a standing station, validating it as a tool for follow-up and screening [6,7] The Formetric 4D tool facilitates clinical practice by analyzing the spinal column. It is completely radiationfree and is a non-invasive method that has been shown to be highly correlated with radiography and is extremely usable and reproducible.

It is essential to have an instrument that provides scientifically valid data for an overall assessment of the patient's posture that can be easily used by nursing and rehabilitation sciences students. The present study aims to evaluate for the first time Formetric 4D [8,9] reliability (internal consistency, intra-rater and inter-rater reliability) in a sitting position, and evaluate its validity comparing the results with the anthropometric measurements (through the Spearman's rho correlation coefficient) detected during the postural evaluation.

\section{$2 \quad$ Material and Methods}

\subsection{Population and procedures}

To conduct this study, individuals were recruited from the spinal unit of the Paraplegic Center in Ostia, Italy between June and September 2018. According to the established inclusion criteria, they needed to be over eighteen years old, have a spinal injury at the lumbar spine level, have no pressure injuries during the study, and use a wheelchair more than $50 \%$ of the time each day. Individuals who had pressure lesions, pregnant women, and patients in a state of entrapment were excluded from the study. As a preliminary step (t0), an interview was carried out with each participant during which every person was specifically informed about the procedures and aims of the study; personal data necessary for the research were collected after the acquisition of informed consent. In the first phase of the study (t1), the participants were evaluated by an occupational therapist through postural assessment. In the second phase ( $\mathrm{t} 2$ ), each individual was evaluated with rasterstereography in a sitting position using the Formetric 4D by three different raters at the Policlinico Umberto I, Rome, Italy. With the assistance of two operators, the user transferred from the wheelchair to 
a stool that was adjustable in height. The same positioning was performed for all subjects. The third phase (T3) consisted of the test-retest, carried out after about four hours: at first, the detection was performed on the same participant three times by three different rater. After four hours, the detection was repeated by the first operator. This made it possible to establish intra-operator reliability between the tests and retests and inter-operator reliability measured on the same participant at the same time by the three different operators.

\subsection{Instruments}

The postural assessment procedures is developed from the ISO 16840:20132018 standards, which permits the determination and recording of a person's posture while seated in a wheelchair; the standard terms and definitions for use in describing both the posture and the anthropometrics of a person seated in a wheelchair. $(3,4)$ The purpose of this assessment procedure is to provide step by step directions to perform assessment tasks, and gather and analyze information when recommending seating and/or mobility equipment. The postural assessment is divided into three parts: observation of the person in current seating system, assessment in the supine position, and recording of anthropometric measurements. Prior to assessing current sitting posture, optimal positioning in the chair is obtained. The position of the participant in a wheelchair is observed to acquire the position of the head, limbs, trunk, and pelvis under normal conditions, that is, how the subject positions him- or herself in his or her daily routine. Subsequently, the participant is asked to lie down on the firm surface in a supine position and to align the body, head and pelvis (to neutral). The mobility of the pelvis is observed; in particular, for this study, the movements of pelvic tilt/ lumbar lordosis, pelvic rotation, and pelvic obliquity were investigated. In order to recorder the anthropometric measurements during the sitting evaluation if the person's pelvis tends to assume a posterior or anterior pelvic tilt / pelvic obliquity / pelvic rotation, and he/she is unable to independently move his/her pelvis to neutral, the flexibility is assessed passively. To perform the rasterstereography, through the Formetric 4D tool (Figure 1), the participant positioned him- or herself for a few seconds two meters from the detection device that uses halogen light projected on the participant's back in the form of a special horizontal line grid; the light is detected by a digital camera.

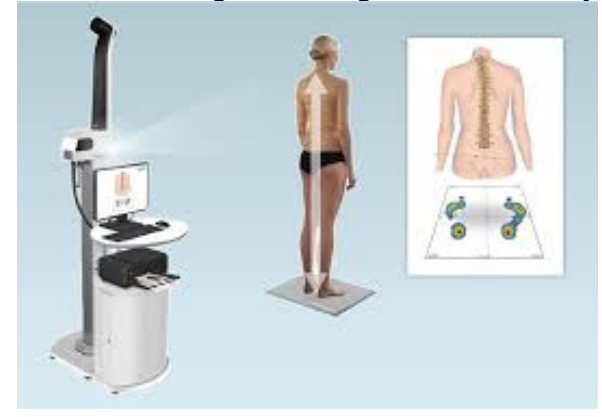

Figure 1 - Formetric 4D tool for rastesterography 
Thanks to this optical scan, the system automatically detects anatomical landmarks, represented by $\mathrm{C} 7$ or prominent cervical vertebra, sacrum and lumbar dimples, and the median or symmetry line. The software analyzes the data thus obtained and reconstructs the shape of the back, the column, and the position of the basin in three dimensions: it creates 12 images in 6 seconds, calculating and representing the average value. Moreover, thanks to the three-dimensional reconstruction, the scan is carried out only on the posterior body surface; therefore, the subject does not have to reposition for the analysis on the other planes, thus minimizing the effect of postural variations.

\subsection{Statistical analysis.}

The statistical analysis of the study results was performed using SPSS software. The internal consistency of the Formetric 4D was measured with Cronbach's alpha. The intraclass correlation coefficient (ICC) was calculated to evaluate the intrarater (the same operator $\mathrm{t} 2 \mathrm{-t} 3$ ) and inter-rater reliability (three different operators $\mathrm{t} 2$ ) of the instrument. The correlation coefficient Spearman's rho was used to measure the correlation between the data obtained with the rasterstereography and the anthropometric measurements detected during the postural evaluation. Statistical significance was established for a $\mathrm{p}$ less than 0.05 .

\section{Results}

Ten participants were recruited according to the established inclusion criteria. All the recruited patients agreed to participate and signed informed consent. Nine subjects (5F-4M) completed the study: their mean (SD) age was 44.22 (12.21), with a mean (SD) hours on wheelchair of 11.67 (2.45). The clinical characteristics can be synthetized as follow: mean (SD) years of injury 23.14 (12.21), lesion level C7 (1), L2 (1), L3 (1), T10 (1), T12 (2), T3 (1), T4 (2).

To assess that the rasterstereography was a reliable tool in evaluating the seated position of the person with spinal cord injury, the Cronbach's alpha was assessed and the tool was found to have an alpha of 0.74 ; intra-observer and inter-observer reliability were $0.91(0.62-0.98)$ and $0.96(0.88-0.99)$, respectively.

These values confirm the stability of the rasterstereography both in the evaluation of the same subject at different times by the same rater between $\mathrm{t} 2$ and $\mathrm{t} 3$ (intra-rater reliability) and in the measurements by three different operators assessed at $\mathrm{t} 2$ (interrater). To evaluate the validity of the rasterstereography, the Formetric $4 \mathrm{D}$ results were correlated with the anthropometric measurements recorded by the operator during the postural assessment. The correlations were evaluated using Spearman's rho; some statistically significant correlations were found, as reported in Table 1.

Table 1 - Gold standard analysis: Spearman's rho correlation between Formetric 4D and anthropometric measures recorded through postural assessment procedure

\begin{tabular}{llcccc} 
Pelvic & Pelvic & Floor- & Trunk & SIAS & $\begin{array}{c}\text { SIPS } \\
\text { Width }\end{array}$ \\
Length & Popliteal & Height & $\begin{array}{c}\text { distance } \\
\text { distance }\end{array}$ \\
\hline
\end{tabular}




\begin{tabular}{|c|c|c|c|c|c|c|}
\hline & {$[\mathrm{Mm}]$} & [Mm] & cable $[\mathrm{Mm}]$ & {$[\mathrm{Mm}]$} & {$[\mathrm{Mm}]$} & [Mm] \\
\hline PI (DL-DR) $\left[^{\circ}\right]$ & 0.07 & 0.26 & 0.16 & 0.03 & 0.26 & 0.12 \\
\hline $\begin{array}{l}\text { PIDLDR[mm] } \\
\text { Twist Emibacini DL- }\end{array}$ & 0.11 & 0.33 & 0.20 & 0.09 & 0.24 & 0.04 \\
\hline $\begin{array}{l}\text { DR }\left[^{\circ}\right] \\
\text { KA Kyphotic Apex }\end{array}$ & 0.65 & 0.35 & 0.33 & 0.28 & 0.23 & 0.11 \\
\hline $\begin{array}{l}\text { (VPDM) }[\mathrm{mm}] \\
\text { ITL Inversion Point }\end{array}$ & 0.32 & 0.02 & $.78^{*}$ & 0.40 & $.73^{*}$ & 0.46 \\
\hline $\begin{array}{l}{[\mathrm{mm}]} \\
\text { Lordotic apex LA }\end{array}$ & 0.59 & 0.05 & 0.29 & 0.13 & $.77^{*}$ & 0.54 \\
\hline $\begin{array}{l}\text { (VPDM) [mm] } \\
\text { ILS Reversal Point }\end{array}$ & 0.11 & 0.41 & 0.43 & 0.05 & 0.64 & 0.19 \\
\hline & 0.46 & 0.30 & 0.38 & 0.17 & 0.64 & 0.11 \\
\hline & 0.44 & $-.73 *$ & 0.17 & 0.08 & 0.08 & 0.25 \\
\hline $\begin{array}{l}\text { gnara) }[\mathrm{mm}] \\
\text { ICT-ITL Angle An- } \\
\text { gle (Max) }\left[^{\circ}\right]\end{array}$ & 0.12 & 0.47 & 0.53 & 0.03 & 0.31 & 0.14 \\
\hline $\begin{array}{l}\text { ITL-ILS (Max) } \\
\text { Surface Rotation }\end{array}$ & 0.17 & 0.37 & 0.07 & 0.60 & 0.15 & 0.18 \\
\hline $\begin{array}{l}(\mathrm{Rms})\left[^{\circ}\right] \\
\text { Surface Rotation }\end{array}$ & 0.17 & 0.10 & 0.22 & 0.28 & 0.12 & 0.29 \\
\hline $\begin{array}{l}\text { (Amplitude) }\left[{ }^{\circ}\right] \\
\text { VPDM lateral devia- }\end{array}$ & 0.23 & 0.20 & 0.11 & 0.10 & 0.38 & 0.04 \\
\hline $\begin{array}{l}\text { tion }(+ \text { Max })[\mathrm{mm}] \\
\text { VPDM lateral devia- }\end{array}$ & 0.38 & 0.31 & 0.44 & 0.40 & 0.21 & 0.22 \\
\hline $\begin{array}{l}\text { tion (-Max) [mm] } \\
\text { VPDM Lateral Devi- } \\
\text { ation (Amplitude) } \\
{[\mathrm{mm}]}\end{array}$ & $-.78^{*}$ & $-.79 *$ & 0.21 & 0.33 & 0.03 & 0.54 \\
\hline
\end{tabular}

* $\mathrm{p}<0.05 ;{ }^{* *} \mathrm{p}<0.01$

Abbreviations: VP = prominent vertebra (spiny apophysis of C7); $\mathbf{D R}$ and $\mathbf{D L}=$ right and left lumbar dimple (Michaelis dimples); DM = average point of the segment that connects DR and DL; ICT = cervicothoracic inversion (cervico-dorsal hinge); ITL = thoraco-lumbar inversion (back-lumbar hinge); ILS = lumbo-sacral inversion (lumbo-sacral hinge); KA = apex kyphosis (max curvature of the dorsal kyphosis); $\mathbf{L A}=$ apex lordosis (max curvature of lumbar lordosis); VP-DM trunk length: VP and DM joining segment length.; DL-DR pelvic inclination: vertical difference between DL and DR.; Lateral deviation VP-DM: on the frontal plane, horizontal lateral deviation of the centers of the vertebral bodies with respect to the joining line VP-DM (rms = quadratic mean, $\max =$ maximum value); Lateral deviation VPDM (+ max): max deviation to the right; Lateral deviation VPDM (-max): max deviation to Sn; Pelvic inclination (dimples): arithmetic mean of the 2 angles formed by the perpendicular to the surface in DR and DL and the vertical axis (mean pelvic torsion); ICT-ITL (max) cytotic angle: upper angle formed by tangents to the sagittal curve in ICT and ITL (represents the maximum value of the cytotic angle); ITL-ILS lordotic angle (max): upper angle formed by tangents to the sagittal curve in ITL and ILS (represents the maximum value of lordotic angle); SIAS distance [Mm]: Antero-superior iliac spinal cord; SIPS distance [Mm] Posterior-superior iliac spine 


\section{Discussion}

This study highlighted the ability of Formetric $4 \mathrm{D}$ to provide consistent results at different times and with different evaluators. Compared to the postural evaluation, the degree of accuracy of the measurements acquired with Formetric 4D, analyzed through Spearman's rho, showed positive correlations with anthropometric measurements, such as the width and length of the pelvis, the distance of the popliteal earth-hollow, trunk height, and the distance of the anterior-superior and posteriorsuperior iliac crests. These correlations are statistically significant, indicating a covariance with the following anatomical measurements: the torsion of emydines, the kyphotic apex, the lordotic apex, and the lordotic angle. However, these correlations currently do not allow for the precise definition of the elements that influence the proportional trend of the variables, but only show the covariance between the data. While other studies in the literature have shown the usability, sensitivity, and repeatability of the Formetric D with participants in an upright position, this pilot study demonstrated the reliability of the Formetric 4D as a tool for the follow-up and screening of this population in a sitting posture for the first time. This study also demonstrated how this tool can complement postural assessments done by occupational therapists, objectifying them. Expressing the anatomic characteristics, postural attitudes and the deformities, heretofore recordered subjectively by the therapist, in a concrete image. The correlation between these two different measurement instruments is an advantage not only at the clinical level, but also at the educational level. In fact, postural assessment is a very difficult assessment for students to learn, because it requires years of experience and specific anatomical knowledge. Accompanying the demonstrations of the practice with images collected technologically through rasterstereography, this method will be of great help in university teaching. Thanks to rasterstereography, in fact, students will be able to have a three-dimensional view of the shape of the back, the spine, and the position of the pelvis in a sitting position and can compare it with the objective examination.

The limited number of samples although it was defined in relation to the type of study can represent a limitation of the study. A larger sample of the population would have allowed greater generalization of the results. Therefore, for future studies on the topic, we hope to increase the sample selection; this could, for example, collect information about subjects with different diagnoses, allowing additional comparisons between the measured variables, and taking into account differences in the population.

\section{Conclusions}

The present study provides information enabling the use of the Formetric 4D tool in clinical, research, and educational settings. In the clinical field, the tool will be useful for occupational therapists in analyzing the postural characteristics of the spine and the pelvis, providing an objectified postural evaluation. This allows therapists to obtain objectively valid data that will be useful for preventing deformities and carrying out postural follow-ups. In the field of research, the Formetric 4D will be useful as it 
provides a starting point for expanding the research for wheelchair posture broadening the research to other diseases or using different support surfaces. And finally, at the educational level, this will be a very useful tool that allows students to have a threedimensional representation of the anatomical components involved in the sitting position, helping them to learn and gain an in-depth understanding of how to perform an objective postural assessment examination.

\section{Compliance with Ethical Standards}

We certify that all applicable institutional and governmental regulations concerning the ethical use of human volunteers were followed during the course of this research.

\section{Conflict of Interest}

All authors declare no conflict of interest.

Founding: None

\section{References}

1. Sonenblum SE, Sprigle SH, Martin JS. Everyday sitting behavior of full-time wheelchair users. J Rehabil Res Dev. 2016;

2. Mattie J, Borisoff J, Miller WC, Noureddin B. Characterizing the community use of an ultralight wheelchair with "on the fly" adjustable seating functions: A pilot study. PLoS One. 2017;

3. International Organization for Standardization. ISO 16840-1:2013 Wheelchair seating -- Part 1: Vocabulary, reference axis convention and measures for body segments, posture and postural support surfaces [Internet]. 2nd ed. 2013. Available from: https://www.iso.org/standard/42064.html

4. International Organization for Standardization. ISO 16840-2:2018 Wheelchair seating -- Part 2: Determination of physical and mechanical characteristics of seat cushions intended to manage tissue integrity [Internet]. 2nd ed. 2018. Available from: https://www.iso.org/standard/66972.html

5. Cohen LJ, Fitzgerald SG, Lane S, Boninger ML, Minkel J, McCue M. Validation of the seating and mobility script concordance test. Assist Technol. 2009;

6. Tabard-Fougère A, Bonnefoy-Mazure A, Hanquinet S, Lascombes $\mathrm{P}$, Armand S, Dayer R. Validity and reliability of spine rasterstereography in patients with adolescent idiopathic scoliosis. Spine (Phila Pa 1976). 2017;

7. Scheidt S, Endreß S, Gesicki M, Hofmann UK. Using video rasterstereography and treadmill gait analysis as a tool for evaluating postoperative outcome after lumbar spinal fusion. Gait Posture. 2018;

8. Padulo J, Ardigò LP. Formetric 4D rasterstereography. BioMed Research International. 2014;

9. Guidetti L, Bonavolontà V, Tito A, Reis VM, Gallotta MC, Baldari C. Intra- and interday reliability of spine rasterstereography. Biomed Res Int. 2013. 